\title{
Artificial intelligence in service of medicine
}

\author{
El-Hassoun $\mathrm{O}^{1}$, Maruscakova L ${ }^{2}$, Valaskova $\mathrm{Z}^{3}$, Bucova $\mathrm{M}^{2}$, Polak $\mathrm{S}^{1}$, Hulin $\mathrm{I}^{4}$ \\ Institute of Histology and Embryology, Faculty of Medicine, Comenius University, Bratislava, Slovakia. \\ olia.elhassoun@fmed.uniba.sk
}

\begin{abstract}
The race to make the dream of artificial intelligence a reality comes parallel with the increasing struggle of health care systems to cope with information overload and translational pressure. It is clear that a shift in the way data is generated requires a shift in the way they are processed. This is where Al comes with great promises to solve the problem of volume versus applicability of information in science.

In medicine, $\mathrm{Al}$ is showing exponential progress in the fields of predictive analysis and image recognition. These promises however, come with an intricate package of ethico-social, scientific and economic implications, towards which a reductionist approach leads to distorted and dramatic predictions. All this, in a time when the growing pressure on healthcare systems towards defensive medicine begs the question of the true need for Al for good medical practice.

This article examines the concept and achievements of $\mathrm{Al}$ and attempts to offer a complex view on the realistic expectations from it in medicine, in the context of current practice (Ref. 38). Text in PDF www.elis.sk.

KEY WORDS: algorithms, artificial intelligence, image recognition, neural networks, predictive analysis.
\end{abstract}

\section{Introduction}

With the rise of modern science, scientists seemed to gravitate towards believing that we have to find the equation for everything in nature (14), aided by heavy influence from physics and engineering which used differential equations do describe dynamic systems (13). Even though such approach did prove useful in prediction and management of certain biological phenomena, equations have not yielded the same success in ecology that they have in physical sciences, suggesting that a new approach is needed (13). Crick explained, that the ultimate goal of the modern movement in biology is to explain all biology in terms of physics and chemistry (15). This current approach, a reductionist one, it ignores fundamental properties of complex systems which are based on interactions between the parts (15). Sugihara concluded, that it is time to abandon the search for equilibrium in the complex, nonlinear systems that nature produces, seductively simple correlations may appear for a period of time, but in a chaotic system such correlations do not provide true insight (13). As science turns to complexity, one must realize that complexity demands attitudes quite different from those common in physics. Each complex system is different,

\footnotetext{
${ }^{1}$ Institute Of Histology and Embryology, Faculty of medicine, Comenius University, Bratislava, Slovakia, ${ }^{2}$ Institute of Immunology, Faculty of Medicine, Comenius University , Bratislava, Slovakia, ${ }^{3}$ Institute of Pathophysiology, Faculty of Medicine, Comenius University, Bratislava, Slovakia, and ${ }^{4}$ Faculty of Medicine, Comenius University, Bratislava, Slovakia

Address for correspondence: El-Hassoun Olia, MD, PhD., Institute Of Histology and Embryology, Faculty of medicine, Comenius University Bratislava, Sasinkova 4, SK-811 04 Bratislava, Slovakia.
}

Acknowledgement: This work was supported by the grant of Ministry of Education of Slovakia VEGA 1/0610/18. apparently there are no general laws for complexity. Instead, one must reach for lessons that might, with insight and understanding be learned in one system and applied to another (16). Bearing this in mind, we wish to address the question of artificial intelligence in medicine. It being a matter with a potential significant impact on the future course of medical, social and economic development. We do not intended to repeat the content of other reviews and introductory articles $(1,9,38)$, but to advance a view from the perspective of a practicing physician addressing fellow physicians and healthcare providers.

\section{Dream of artificial intelligence}

The dream of artificial intelligence is not a new trend. Attempts to make an autonomous humanoid date back to the third century in China (1). The term AI was first coined by McCarthy in 1955 as the science and engineering of making intelligent machines. Contemporary developing AI is a branch of engineering integrated into many scientific areas (2). Over the years this term has grown to be overused and misused to include all kinds of computerized automated systems, including logical programming, probability algorithms and remote-controlled surgical robotics. The general premise is that these systems are capable of processing data from a large database and mimic human decisions based on a specific set of instructions. Many of these systems however don't implement a learning behavior; that is the ability to perform tasks which are not explicitly programmed (11). In this article, we will focus on AI in terms of basic machine learning and deep learning. These modalities are often used in conjuncture to create various functions of a given application. Deep learning systems are designated deep learning architectures. One of the most successful 
prototypes, used in various medical applications are convoluted neural networks (CNN).

Supervised vs unsupervised learning is a major difference between humans and machines. As the name implies, in supervised learning, data scientists teach the algorithm what conclusions it should reach (19). In unsupervised learning, the software is fed raw data and must figure things out for itself without human help. Today's most practical machine learning uses supervised learning, which limits how far artificial intelligence can go $(17,18)$. Although Googles cat experiment, which was based on unsupervised learning, seemed in some way to be a dead end, many agree that unsupervised learning will be a very important component in building intelligent systems (17).

A machine learning system is trained by introducing data to its learning algorithms, from which it uncovers patterns, builds models and makes predictions based on the best fit model. The key factor in model building by machines relies on data features which are manually designed by field experts. The identification of features for each task still remains a challenge. Similarly, when processing raw data, proper encoding is a main limitation. Deep learning is advancing from this by being able to independently discover effective features and their mappings from data $(9,35)$. So far, these complex networks are able to discover previously unknown highly abstract patterns and correlations from multi-dimensional data, which will provide insight to better understand their nature (9).

\section{Healthcare struggle}

Medicine, as a complex scientific discipline is constantly challenged by problems of the acquiring, processing and application of large amount of knowledge (2). These struggles partially emerged as a consequence of the integration and use of methods which produce big data $(3,34)$. In addition, there are constant expectations such as improving access to services for patients, reducing operating costs and improving treatment outcomes (5). The burden of adapting the workflow to ever changing norms and guidelines increases the frustration of healthcare workers and forces highly trained specialists to spend more and more hours on paperwork instead of focusing on patient care. The severe shortage in healthcare professionals is also due to the vast increase in indicated medical procedures and examinations. For example, the number of image studies performed has sky-rocketed over the last two decades (12).

With such burden on healthcare systems, AI comes with a handful of promises and has demonstrated the potential to solve them. Bioinformatics dedicated to the transformation of big data into valuable knowledge has rapidly advanced since the 2000s. With careful attention from the industry, companies are competing to develop effective healthcare technologies. Examples include IBM's Watson oncology platform, and Google's deep mind health (9).

\section{Predictive analysis}

The shift from descriptive analysis to predictive analysis itself is a great jump in the way we perceive healthcare and what are our expectations from it. Market dynamics geared the motivation of industries to provide more reliable plans and budgets. The current situation of healthcare as an industry makes it no different. Electronic medical records are a source of big data which provide a tremendous amount of information extracted via various deep mining and deep learning techniques $(33,35)$. Through predictive analysis, AI is expected to pave the path towards precision medicine. For instance; in cardiovascular medicine several machine learning algorithms have been developed to improve patient care, cost-effectiveness and reduce readmission and mortality rates (20). Machine learning proved superior to statistical methods in the field of cancer risk and recurrence prediction, with different level of performance based on the used architecture and the type of input data (28). A reasoning system based on mediative fuzzy logic was proposed for predictive diagnosis of heart disease. This system is able to process incomplete, uncertain and inconsistent information (27). With such abilities, AI will ease the transition from evidence based medicine to personalized medicine, by reducing the bias resulting from restricting parameters preventing patients from being included in large studies. A patients own record history can create the substrate for personalized predictions, as seen in (32).

\section{Image analysis}

In recent years, image recognition using machine learning has rapidly improved and is being tested and applied to clinical imaging in various fields, examples include gastroscopy imaging, skin cancer classification, radiation oncology, diabetic retinopathy, histologic classification of different types of biopsies, mammograms, CT and MRI images and colocytoscopy of colorectal lesions (8, 29, 37). Convoluted neural networks are architectures which proved very useful in medical imaging. As we mentioned earlier, most practical applications use supervised learning for the building of different models. For image analysis, the key tasks are anomaly classification, structure segmentation and recognition, all of which are performed by various types of neural networks (9).

Machine learning applications in medical imaging are being successfully implemented in the field of radiotherapy. Applications include the construction of synthetic CT from MRI (24), various decision support and treatment recommendations, target volume delineation and dose prediction (25). These architectures can absorb a lot of information in the module and come up with multiple plans. Princess Margaret cancer center in Canada has been applying automated planning since 2009 (30). The automated system requires patient $\mathrm{CT}$ and contours as input and delivers plans generated in minutes. Training a neural network for organ segmentation takes typically 50 to 100 patients, although there is no optimal figure. For this task, the more important is representative rather than volume of entries to avoid bias. If the training data are insufficient they will of course affect the quality of results. Finding patient matches to construct a module is always a challenge, including a larger number of patients will increase variability. One must be rigorous in choice of data which has to be similar to be included in the module; for example, hip implant patients are excluded from a module for prostate cancer therapy planning (31). 
Many of the clinically integrated systems nowadays are validated by independent reviews of specialists. It is important to understand that not everything will be automated. These are methods for knowledge transfer and making treatment more consistent, which will also help liberate human resources to focus on other problems. Moreover, building modules should not require approval of an ethical committee because real patient data are not identifiable, by which the issue of privacy is solved.

Different architectures are being developed not only to takeover a task performed by humans, but also to detect and evaluate factors which cannot be appreciated by physicians. An example are sub-visual image features in histological slides (36), these include segmentation of nuclei, cytoplasm and stroma. Such fine features which are not detectable in routine light microscopy are expected to be of diagnostic, prognostic and predictive value.

\section{Limitations}

In general, most of the image analysis architectures were able to solve a given task at a comparable, sometimes even a better success rate than a clinician. But if we bear in mind the complex workflow of the diagnosis and treatment process, the application of AI in everyday practice is still in its cradle. For a physician who needs to define several different parameters to make a diagnosis of a certain cancer, having to spend time contouring an image so the machine will give a supposedly more accurate evaluation of only one or a few parameters, such as mitotic count is perceived by physicians more like a burden than it is real help. AI for endoscopy still requires high quality images and any of the common artefacts can cause diagnostic errors (8). Images still need to be selected and contoured by an experienced clinician. It seems misleading to brag about how fast an AI system can evaluate a great number of images in a short time, when they were already previously selected and contoured in a manual time consuming fashion.

Several institutes are racing to build AI based classifiers which are able to produce a diagnosis from scanned histological slides. In most cases, the machine will provide an answer within a prediction score; e.g. there is a $90 \%$ chance that this tumor is a glioblastoma. This is only possible when applied in a specific context. The machine will not be able to recognize what it was not trained to see. In this regard, diagnostic complexity remains a challenge for any so far developed classifier. A diagnosis in pathology is based on knowledge and experience, but with high correlation between histomorphology and clinical context. Likewise, the strength of a radiologist lies not in the ability to detect and classify clinical images, but in the ability to make clinical judgements based on these data. This knowledge comes not only from radiology practice, but also from many years of undergraduate training (12). Discordance and interobserver variability in the interpretation of images usually do not occur in cases with clear cut diagnoses, but rather in gray zone lesions which do not meet standardized diagnostic criteria. A certain sort of inter-algorithm variability will occur as a result of various variables in the system. As an example; the effect of pre-processing strategy on correct classification rate is demonstrated in (29).
If we are to evaluate how we expect artificial intelligence to solve our problems we must first examine the methods with which we acquire knowledge. In science and medicine, a great deal of new knowledge is generated through analyzing past event, i.e. retrospective/descriptive analysis. This is the type of analysis which asks the question: what happened? And uses traditional statistics to help understand past events and how they might influence future outcomes (21). If what scientists do is study past events and make hypotheses about the future, then the insights we acquire are a matter of probability, not certainty (4). We wish not to dip into the philosophy of uncertainty but only to emphasize that if our decision is mathematically calculable then it is easily produced by an "accurate machine". If things were that simple, then IBM's Watson oncology platform would have achieved revolutionary success. Doctors were not surprised that IBM claims did not pan out, instead it reassured their views of the flaws of automated systems. Reinforcing the fact that the task of providing healthcare is far more complex than a rigid diagnostic and treatment algorithm, and that the life of a patient can never be reduced to a statistical number. Still, the public opinion is an easy prey to media fabrications. This is why it is important to elucidate certain concepts from misconceptions (26), and define where we really stand on the continuum of technological development.

Intelligence is only one factor in progress; it produces thoughts which require further conducting of experiments, building prototypes, fighting through the limitations of simulation and undergoing validation. All are processes which must happen in real time and cannot be sped up (10), guaranteeing it to take some time before it can offer ready-to-use solutions. The focus on intelligence as the basis of progress ignores the fact that we as humans engage with evolutionary pressure using our entire bodies (22). This means that our information processing is the product of mind-body perception and interaction. This and other components of human cognition are usually eliminated in algorithmic simulation of mental functions as we see in AI, which definitely impedes the emergent effect of our human decision making.

In everyday practice, various clinical situations require the integration of knowledge from different sources. Knowledge of pathophysiology mechanisms cannot answer all clinical questions but it is still useful in many circumstances where outcome studies might be lacking, incomplete, or invalid. Physicians will always need the intellectual flexibility to integrate the several forms of knowledge with clinical reasoning to provide the very best patient care (23). Competent professionals will find it very difficult to "trust" the decision of a machine.

\section{Implications}

AI promotes its potential contributions to healthcare with emphasis on the elimination of error caused by the human factor. According to the US Centers for Disease Control and Prevention, patient harm is the third highest cause of health-related mortality (6). But as we explained earlier, no system available so far can be fully automated, let alone a complete healthcare unit. In general, we rely on the human factor for many crucial tasks such as data 
entry and certain stages of sample manipulation. Many clinically significant patient data are obtained through taking the patient's history. This process alone requires meticulous care, bearing in mind the patient's psychological status, memory, orientation, and general tendencies towards certain behavior like alcohol consumption. All are factors which will influence the possibility or willingness of the patient to provide information. Trust is the keystone of the physician-patient relationship, and it is established through interpersonal communication (7), something which is very difficult to imagine be replaced by a chatbot.

Modern healthcare is ill through several mechanisms. The valuable physician-patient time is being compromised by increased workload on physicians, forcing them to resort to methods of lesser quality such as patient history forms. Media portrayal and idealistic patient expectations fueling a flawed judicial system are creating immense pressure on physicians to be errorless, leading them to enlarge their list of required tests and examinations to be "covered just in case”, burdening the system even further. AI's promise of automation of some procedures can help liberate human resources to focus on other problems. Which in the context of a defensive approach could aggravate the problem and deepen the financial load. Creating diagnostic classifiers might also help set a fixed range for a healthier expectation of diagnostic accuracy. This however is a double edged sword, the use of AI in decision making could also cause further dissolving of responsibility in the already plagued system.

The huge hole in healthcare systems is caused by being profit geared, as opposed to what is best for the population. Analysis tools are designed to identify "costly populations" and other parameters which can be translated into expenses and investments. Back here in the real world, physicians and scientists are still facing restrictions in the accessibility of data, information and medical publications. Physicians have to work through the constant pressure of lack of finances, while at the same time, a ridiculous amount of money is being allocated to AI research, aside from separate programs run by large private companies. AI is advertised as a method to deliver top class treatment to poor areas, while AI products are already being sold for unreasonable prices, to the chosen few who can afford it. For overworked professionals, any change in workflow is understandably perceived as undesirable, and many don't believe in a true possibility of artificial intelligence when are in a constant struggle with the artificial stupidity of integrated platforms compulsory for everyday practice.

\section{Concluding remarks}

The pursuit of creation of AI was not driven by modern age pressure on healthcare systems. Still, healthcare can benefit greatly if AI technologies are directed towards solving its problems. Breakthrough is possible when we are able to define our healthcare problems in its complex context, be self-critical and allow for a change. If the incentive for building AI medical support is the claim that the human brain has limited capacity, then increasing the number of parameters and creating a machine that can speedily put them together sounds like a good idea gone astray. The rapid development of data acquiring technologies necessitates a change in our approach to problem solving. Perhaps AI will help us create a new view of how to address healthcare issues.

Achievements of AI can be appreciated but only within certain limits. AI is insufficient in its lacking of sense and emotion, the effect of which is an incalculable integral part of our decision making. In the medical field, a patient can have resting heart rate at 50 per minute, and at rate 70 per minute will subjectively begin to feel tachycardic. Indeed a good physician decides upon the patients treatment based on knowledge and experience, but also in part using his intuition; the physician can choose to administer a lower dose of a certain medication, albeit has no explanation for it. Ultimately, medicine is also about the patients trust in the physician; a patient who trusts and believes their doctor has more hope for recovery (38). These parameters are just more difficult to incorporate in an AI decision system. The subconscious component of decision making in cases sharing a similar clinical course is still inadequate in AI. Eventually, for the physician, making the right decision is worth more than winning the lottery. The modifier "artificial" provides a defense mechanism against this type of intelligence, indicating it's inanimate and only-calculated nature. Human intelligence will probably remain as the good base and artificial intelligence the augmentation. This also applies when we count in human experience which is hardly programmable as it lacks precise calculation.

\section{References}

1. Hamet P, Tremblay J. Artificial intelligence in medicine. Metabolism 2017; 69S: S36-S40. doi: 10.1016/j.metabol.2017.01.011.

2. Sniecinski I, Seghatchian J. Artificial intelligence: A joint narrative on potential use in pediatric stem and immune cell therapies and regenerative medicine. Transfus Apher Sci 2018: S1473-0502(18)30172-1. doi: 10.1016/j.transci.2018.05.004.

3. Mezencev R. The Future of Biomedical Science. In: Hulín I, Ostatníková D, Mezencev R et al. On the Scientific Observation in Medicine. Bratislava, Slovakia: AEPress, Ltd.; 2015.

4. Baiju NT. The Metaphysics of Big Data: The Problem of Induction. [online] Big Data Made Simple. Available at: https: //bigdata-madesimple. com/the-metaphysics-of-big-data-the-problem-of-induction/ [Accessed 24 Nov 2018].

5. Ai.intel.com. 2018. [online] Available at: https: //ai.intel.com/nervana/ wp-content/uploads/sites/53/2018/05/Predictive-Analytics-in-Healthcare. pdf [Accessed 5 Nov 2018].

6. Singh R, Singh A, Singh DR, Singh G. Improvement of workflow and processes to ease and enrich meaningful use of health information technology. Adv Med Educ Pract 2013; 4: 231.

7. Pellegrini CA. Trust: the keystone of the physician-patient relationship. 2017. Retrieved from bulletin. facs. org/2017/01/trust-the-keystoneof-the-physician-patientrelationship/\#. WsRaldPwaCQ.

8. Hirasawa T, Aoyama K, Tanimoto T, Ishihara S, Shichijo S, Ozawa T et al. Application of artificial intelligence using a convolutional neural network for detecting gastric cancer in endoscopic images. Gastric Cancer 2018; 21 (4): 653-660.

9. Min S, Lee B, Yoon S. Deep learning in bioinformatics. Briefings Bioinform 2017; 18 (5): 851-869. 
10. Kelly K. The Myth of a Superhuman AI | Backchannel. 2017. online WIRED. Available at: https: //www.wired.com/2017/04/the-myth-of-asuperhuman-ai/ [Accessed 6 Jul 2018].

11. Watson Health. Artificial intelligence, machine learning and deep learning: What do they mean? - Watson Health Perspectives. [online] Watson Health Perspectives. Available at: https: //www.ibm.com/blogs/watsonhealth/what-do-they-mean/ [Accessed 11 Jul 2018].Liew, C. (2018). The future of radiology augmented with Artificial Intelligence: A strategy for success. Eur J Radiol 2018; 102: 152-156.

12. Popkin G. Is It Foolish to Model Nature's Complexity With Equations? 2015. online WIRED. Available at: https: //www.wired.com/2015/10/ is-it-foolish-to-model-natures-complexity-with-equations/ [Accessed 15 Sep 2018].

13. Stewart I. Does God play dice?: The new mathematics of chaos. Penguin UK, 1997.

14. Pernick N. Focusing on Preinvasive Neoplasia. Arch Pathol Labor Med 2018; 142 (5): 558-558.

15. Goldenfeld N, Kadanoff LP. Simple lessons from complexity. Science 1996; 284 (5411): 87-89.

16. Simonite T. The Missing Link of Artificial Intelligence. 2016. online MIT Technology Review. Available at: https: //www.technologyreview. com/s/600819/the-missing-link-of-artificial-intelligence/ [Accessed 16 Sep 2018].

17. Brownlee J. Supervised and Unsupervised Machine Learning Algorithms. 2016. online Machine Learning Mastery. Available at: https: // machinelearningmastery.com/supervised-and-unsupervised-machinelearning-algorithms/ [Accessed 22 Sep 2018].

18. Castle N. Supervised vs. Unsupervised Machine Learning. 2017. online Datascience.com. Available at: https: //www.datascience.com/blog/ supervised-and-unsupervised-machine-learning-algorithms [Accessed 7 Nov 2018].

19. Krittanawong C, Zhang H, Wang Z, Aydar M, Kitai T. Artificial intelligence in precision cardiovascular medicine. J Amer Coll Cardiol 2017; 69 (21): 2657-2664.

20. Halo. (n.d.). Descriptive, Predictive, and Prescriptive Analytics Explained. [online] Available at: https: //halobi.com/blog/descriptive-predictive-and-prescriptive-analytics-explained/ [Accessed 17 Nov 2018].

21. Stern E. Embodied cognition: A grasp on human thinking. Nature 2015; 524 (7564): 158.

22. Kyriacou DN. Evidence-based medical decision making: deductive versus inductive logical thinking. Acad Emerg Med 2004; 11 (6): 670-671.

23. Han X. MR-based synthetic CT generation using a deep convolutional neural network method. Med Phys 2017; 44 (4): 1408-1419.
24. Berlin A, Conroy L, Tjong MC, Craig T, Chung P, McIntosh C, Purdie TG. Clinical Application of a Novel Voxel-and Machine LearningBased Automated Planning Method for Prostate Volumetric Arc Radiation Therapy. Internat J Radiat Oncol Biol Phys 2018; 102 (3): e533.

25. Stoeklé HC, Charlier P, Hervé C, Deleuze JF, Vogt G. Artificial intelligence in internal medicine: Between science and pseudoscience. Eur J Intern Med 2018; 51: e33-e34.

26. Iancu I. Heart disease diagnosis based on mediative fuzzy logic. Artificial intelligence Med 2018.

27. Richter AN, Khoshgoftaar TM. A review of statistical and machine learning methods for modeling cancer risk using structured clinical data. Artificial intelligence Med 2018.

28. Gandomkar Z, Brennan PC, Mello-Thoms C. MuDeRN: Multi-category classification of breast histopathological image using deep residual networks. Artificial intelligence Med 2018.

29. Purdie TG, Dinniwell RE, Fyles A, Sharpe MB. Automation and intensity modulated radiation therapy for individualized high-quality tangent breast treatment plans. Internat J Radiat Oncol Biol Phys 2014; 90 (3): 688-695.

30. Buschmann M, Sharfo AWM, Penninkhof J, Seppenwoolde Y, Goldner G, Georg D et al. Automated volumetric modulated arc therapy planning for whole pelvic prostate radiotherapy. Strahlenther Onkol 2018; 194 (4): 333-342.

31. Kang S. Personalized prediction of drug efficacy for diabetes treatment via patient-level sequential modeling with neural networks. Artificial intelligence 2018; 85: 1-6.

32. Bates DW, Saria S, Ohno-Machado L, Shah A, Escobar G. Big data in health care: using analytics to identify and manage high-risk and highcost patients. Health Affairs 2014; 33 (7): 1123-1131.

33. Yang CC, Veltri P. Intelligent healthcare informatics in big data era. Artificial intelligence Med 2015; 65 (2): 75-77.

34. Lan K, Wang DT, Fong S, Liu LS, Wong KK, Dey N. A survey of data mining and deep learning in bioinformatics. J Med System 2018; 42 (8): 139.

35. Liszewski K. Big Data from Images of Tiny Tissue Samples. Genet Engin Biotechnol News 2014; 37 (16): 1-26.

36. Tang Q, Liu Y, Liu H. Medical image classification via multiscale representation learning. Artificial intelligence Med 2017; 79: 71-78.

37. Kononenko I. Machine learning for medical diagnosis: history, state of the art and perspective. Artificial Intelligence 2001; 23 (1): 89-109.

38. Raison C. The healing power of the doctor-patient bond. 2011. online Thechart.blogs.cnn.com. Available at: http: //thechart.blogs.cnn. com/2011/05/03/the-healing-power-of-the-doctor-patient-bond/[Accessed 9 Dec 2018]. 